\title{
Toward (Hybrid) Navigation of a Fluorescence Camera in an Open Surgery Setting
}

\author{
Gijs H. KleinJan ${ }^{1,2}$, Nynke S. van den Berg ${ }^{1,3}$, Matthias N. van Oosterom ${ }^{1}$, Thomas Wendler ${ }^{4,5}$, Mitsuharu Miwa ${ }^{6}$, \\ Axel Bex ${ }^{3}$, Kees Hendricksen ${ }^{3}$, Simon Horenblas ${ }^{3}$, and Fijs W.B. van Leeuwen ${ }^{1,3}$ \\ ${ }^{I}$ Interventional Molecular Imaging Laboratory, Department of Radiology, Leiden University Medical Center, Leiden, The \\ Netherlands; ${ }^{2}$ Department of Nuclear Medicine, The Netherlands Cancer Institute-Antoni van Leeuwenhoek Hospital, Amsterdam, \\ The Netherlands; ${ }^{3}$ Department of Urology, The Netherlands Cancer Institute-Antoni van Leeuwenhoek Hospital, Amsterdam, The \\ Netherlands; ${ }^{4}$ Institut für Informatik, Computer Aided Medical Procedures (CAMP), Technische Universität München, Munich, \\ Germany; ${ }^{5}$ SurgicEye GmbH, Munich, Germany; and ${ }^{6}$ Business Incubator, Development Center, Hamamatsu Photonics K.K., \\ Hamamatsu City, Japan
}

\begin{abstract}
With the introduction of the hybrid tracer indocyanine green (ICG)-99mTc-nanocolloid, a direct relation between preoperative imaging and intraoperative fluorescence guidance was established. However, fluorescence guidance remains limited by its superficial nature. This study evaluated the feasibility of a nuclear medicinebased navigation concept that allowed intraoperative positioning of a fluorescence camera $(\mathrm{FC})$ in the vicinity of preoperatively defined ICG-99mTc-nanocolloid containing sentinel nodes (SNs). Methods: Five patients with penile cancer scheduled for SN biopsy were injected with ICG-99mTc-nanocolloid followed by preoperative SPECT/CT imaging. The navigation device was used to provide a real-time augmented reality overlay of the SPECT/CT images and video output of the FC. This overlay was then used for FC navigation. Results: SPECT/CT identified 13 SNs in 9 groins. FC navigation was successful for all 12 intraoperatively evaluated SNs (average error, $8.8 \mathrm{~mm}$; range, $0-20 \mathrm{~mm}$ ). Conclusion: This study reveals the potential benefits of $\mathrm{FC}$ navigation during open surgery procedures.
\end{abstract}

Key Words: augmented reality; sentinel node biopsy; navigation; fluorescence imaging; SPECT/CT

J Nucl Med 2016; 57:1650-1653

DOI: 10.2967/jnumed.115.171645

\section{A}

lthough interventional guidance can be provided using multiple modalities, because of its ability to provide detailed and realtime guidance, fluorescence imaging (FI) is receiving increasing interest. FI is highly effective in superficial applications at known locations but has a limited in-depth potential (1). Radioguidance technologies on the other hand have been shown to have a great in-depth potential and even allow for preoperative confirmation of tracer uptake using, for example, SPECT combined with CT (2).

When separate approaches are used to provide pre- and intraoperative guidance, information may get lost between diagnostic

Received Dec. 22, 2015; revision accepted Apr. 11, 2016.

For correspondence contact: Fijs W. B. van Leeuwen, Interventional Molecular Imaging Laboratory, Department of Radiology, C2-S zone, Leiden University Medical Center, Albinusdreef 2, 2300 RC Leiden, The Netherlands.

E-mail: F.W.B.van_Leeuwen@lumc.nl

Published online May 26, 2016.

COPYRIGHT (C 2016 by the Society of Nuclear Medicine and Molecular Imaging, Inc. imaging and the operation theater. Discrepancies in findings may subsequently result in over- or undertreatment of the patient. To solve these translational issues, and to enable a best-of-both-worlds scenario, a hybrid surgical guidance concept is advocated. This concept is based on integrating multiple complementary features that are of value for preoperative imaging (nuclear medicine) and intraoperative (fluorescence) image-guided surgery (3). This can, in part, be realized using a hybrid tracer that contains 2 distinct signals, for example, the sentinel node ( $\mathrm{SN}$ ) tracer indocyanine green (ICG)- ${ }^{99 m}$ Tc-nanocolloid (1). Recently, this hybrid concept was expanded beyond the field of tracer development to hardware-based integration of radioguidance and FI modalities $(4,5)$.

Surgical navigation using 3-dimensional (3D) nuclear medicine findings has been used to provide directional movement to the lesion of interest, in the form of in-depth information. This technology was considered particularly valuable during SN biopsy procedures (6-8). Apart from navigation of a $\gamma$-probe, the navigation setup has also been used for laparoscope (4), $\gamma$-camera (7), and ultrasound probe (9) positioning. We thus reasoned it should be possible to also navigate a fluorescence camera (FC) in an open surgery setting (Fig. 1).

For penile cancer SN biopsy, we have previously demonstrated that ICG-99m ${ }^{9} \mathrm{C}$-nanocolloid provides superior optical guidance toward SNs compared with blue dye (10). However, in patients with increased fatty tissue surrounding the SNs, the limited tissue penetration of the fluorescent signal was not always sufficient to optically identify the SNs via fluorescence guidance (10). Although the conventional radioguided approach was effective in identifying these SNs, these modalities provided no depth information to the target. By integrating navigation into the procedure, we made an attempt to overcome these shortcomings for penile cancer patients who were to undergo a SN biopsy procedure.

\section{MATERIALS AND METHODS}

\section{Patients}

Five patients with cT1-2N0Mx penile cancer (11) scheduled for SN biopsy and subsequent treatment of the primary tumor were prospectively included. All included groins were clinically node-negative (10).

The institutional review board of The Netherlands Cancer InstituteAntoni van Leeuwenhoek approved this study, and all subjects signed a written informed consent form. Procedures followed were in accordance with the ethical standards of the responsible committee on human experimentation (institutional and national) and with the Helsinki Declaration of 1975 , as revised in 2008 . 


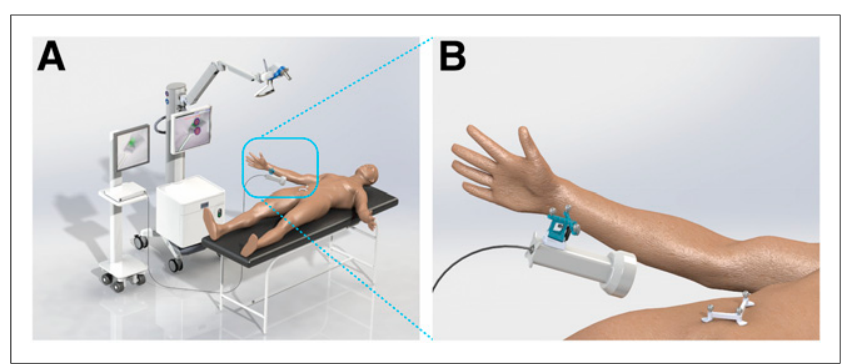

FIGURE 1. Navigation setup. (A) Setup in the operation theater. (B) $A$ reference tracker is placed on both the patient and the FC. Consequently, the navigation device is able to localize the $\mathrm{FC}$ with respect to the position of the patient and the overlayed SPECT/CT images.

\section{Hybrid Tracer Preparation, Injection, and Preoperative SN Mapping and Histopathology}

The hybrid tracer is formed by mixing ${ }^{99 \mathrm{~m}} \mathrm{Tc}$-nanocolloid with a small amount of ICG. Briefly, $2 \mathrm{~mL}$ of pertechnetate $(\sim 1,400 \mathrm{MBq})$ in saline was added to a commercial vial of nanocolloid (containing $0.5 \mathrm{mg}$ of albumin colloid [GE Healthcare]). After incubation at room temperature $(30 \mathrm{~min}$ ) and removal of any excess reactive elements, to the ${ }^{99 \mathrm{~m} T c-n a n o c o l l o i d ~ s o l u t i o n ~(p H ~ 6-7) ~} 0.25 \mathrm{mg}$ of ICG was added to form ICG-99m Tc-nanocolloid $(12,13)$.

For the $\mathrm{SN}$ biopsy procedure of penile cancer, the hybrid tracer injection procedure, preoperative $\mathrm{SN}$ mapping, and histopathologic tissue analysis have been previously described in detail by Brouwer et al. (10). On average $96.7 \mathrm{MBq}$ of ICG- ${ }^{99 \mathrm{~m}} \mathrm{Tc}$-nancolloid was injected in 4 depots around the tumor (Supplemental Table 1 [supplemental materials are available at http://jnm.snmjournals.org]).

\section{Specifications of Navigation Device and FC (Software and Hardware)}

Hardware and Reference Trackers. As navigation device, the declipseSPECT (SurgicEye), in which DICOM files of the preoperatively acquired SPECT/CT datasets can be loaded, was used $(4,8)$.

Preoperative images were acquired with a reference tracker containing three fiducials (hereafter referred to as $\mathrm{RT}_{\text {patient }}$ ) placed on the patient (Fig. 1). In 4 patients, the patient reference tracker $\left(\mathrm{RT}_{\text {patient }}\right)$ was placed on the pubic bone, whereas in 1 patient (patient 2) this was impossible because of the patient's obesity; here the tracker was placed on the superior anterior iliac bone.

The preoperative $\mathrm{RT}_{\text {patient }}$ location was marked (with ink), and during the operation a sterile $\mathrm{RT}_{\text {patient }}$ was placed on the same skin-marked location, allowing the navigation system to spatially merge the preoperative acquired SPECT/CT with the position of the patient in the operating theater (registration). When fused with the real-time white light videofeed generated by the navigation device-embedded overhead camera, different on-screen mixed/augmented-reality views could be created. Intraoperative replacement of the sterile $\mathrm{RT}_{\text {patient }}$ did not negatively affect the surgical workflow.

A second 3-fiducial reference tracker (hereafter referred to as $\mathrm{RT}_{\text {fluorescence }}$ ) was attached to the modified PhotoDynamicEye (m-PDE; Hamamatsu Photonics KK) FC to allow for its tracking by the navigation system (Fig. 1). This FC has the ability to switch between white light and near-infrared FI mode and works under ambient light conditions (15). For easy differentiation between the $\mathrm{RT}_{\text {fluorescence }}$ and the $\mathrm{RT}_{\text {patient }}$, the trackers were geometrically differently shaped.

Incorporation of Imaging Information of FC into Navigation Device. To import the video-feed of the FC into the navigation system, the Bayonet-Neill-Concelman connector (BNC) video output of the FC was taken and converted with a BNC to video graphics array converter (Ligawo), after which the signal was recorded in a Epiphan DVI2PCIe framegrabber (Epiphan Systems Inc.) that was integrated in the navigation device. Customized declipseSPECT 6.0 software (SurgicEye) was used to incorporate the external FC video-feed in both the calibration and the navigation workflow.

Calibration of the FC was performed in the following 2 steps. For intrinsic (e.g., focal length, principle point, lens distortions) and extrinsic camera parameters (translation and rotation with respect to the navigation device reference coordinate system), calibrations were performed using a tracked $7 \times 8$ checkerboard and an open source computer vision library for camera calibration based on the work as described by Zhang (14). These calibrations were performed to allow the augmented projection of the preoperative SPECT/CT data in the FC video image. In the second step, calibration of the transformation between the camera lenses and the $\mathrm{RT}_{\text {fluorescence }}$ on the $\mathrm{FC}$ was performed using a dedicated calibrator reference target. This calibration makes it possible to show the distance from the camera lenses relative to the designated navigation targets found in the SPECT imaging data, in this case the SNs.

To allow the FC to be tracked and navigated in the preoperative SPECT/CT images, a navigation display similar to the one generated for the conventional $\gamma$-probe was used (8). Herein, an overlay of the 3D SPECT/CT images onto the patient was displayed in the videofeed of the FC. This same view also showed the distance from the tip of the FC to the designated targets (SNs).

Integration of the SPECT/CT images with the real-time fluorescence images of the FC could be switched on and off at will. Moreover, because the FC allows for both white light and near-infrared FI, navigation could be performed in both imaging modes. When sudden movements were made with the FC during the navigation procedure, the SPECT/CT overlay followed with a slight lag $(\sim 20-40 \mathrm{~ms})$. This could be corrected easily by holding the FC still for a fraction of a second.

\section{Surgical Procedure: Intraoperative Navigation to SNs}

Before incision, the $\mathrm{FC}$ was navigated in the preoperatively acquired SPECT/CT images toward the SNs in the groin. During this process, the distance between the FC and the center of the $\mathrm{SN}$ was provided. If the $\mathrm{SN}$ was not visible through the skin via FI, the $\gamma$-probe (Neoprobe; Johnson \& Johnson Medical) was used to determine the site for incision.

After incision, alternating $\gamma$-tracing and FI allowed localization of the $\mathrm{SNs}$ in the groin. On localization of the SN, the FC was again navigated to determine the correlation between the acquired fluorescence image and the projected SPECT/CT image. The error of navigation (in the coronal plane) was determined by measuring the distance between the center of the radioactive hotspot and the center of the fluorescence hotspot as seen on-screen from the perspective of the FC.

\section{Light Spectra Measurements}

Light spectra of the FC and the optical tracking device of the navigation device were measured using a Jobin Yvon VS140 linear array fiber spectrometer (Horiba) in the 300- to 1,200-nm range, with an integration time of $0.1 \mathrm{~ms}$. The fiber was held at an approximate $2-\mathrm{m}$ distance from the head of the device of which the light spectra were measured.

\section{RESULTS}

\section{Preoperative SN Mapping}

Preoperative imaging revealed bilateral drainage in 4 of the 5 included patients. In the remaining patient, only unilateral drainage was seen. With SPECT/CT imaging, 13 SNs, dispersed over 9 groins, were identified, of which $30.8 \%$ could already be visualized on the lymphoscintigrams acquired $15 \mathrm{~min}$ after injection of the hybrid tracer (Supplemental Table 1).

\section{Navigation}

In the navigation setup, the overlay of the SPECT/CT images and the FC video-feed was displayed on-screen (Figs. 2 and 3). 
Intraoperatively, 12 of the 13 SNs seen on preoperative imaging were navigated to with the FC (Supplemental Video 1) with an average navigation error of $8.8 \pm 9.1 \mathrm{~mm}$ (median, $7.5 \mathrm{~mm}$; range, $0-20 \mathrm{~mm}$ ). The $1 \mathrm{SN}$ that was resected without using navigation was omitted because of logistical reasons.

Intraoperative repositioning of the $\mathrm{RT}_{\text {patient }}$ directly resulted in a large navigation error (Supplemental Table 1, patients 3 and 4). In patient 4 only, we were able to correct this $\mathrm{RT}_{\text {patient }}$ placement using the preoperative placed skin markings, thereby reducing the navigation error for the other SNs of this patient to $0 \mathrm{~mm}$ (Supplemental Table 1).

In 2 patients (patients 2 and 3), on localization of the SN we experienced that the hotspot in the preoperative images contained multiple (clustered) SNs, resulting in the intraoperative identification of 2 additional SNs.

Overall, the navigation procedure was not found to be of influence on the length of the operation (average operation time, $91 \mathrm{~min}$, including the primary tumor treatment).

Spectral Overlap Between Near-Infrared Camera and Fluorescence Emission of ICG. Optical tracking by the navigation device occurred via reflection of pulsed near-infrared light $(20 \mathrm{~Hz})$ emitted by the tracking head of the declipseSPECT system. Following light reflection on the fiducials present on the $\mathrm{RT}_{\text {patient }}$ and $\mathrm{RT}_{\text {fluorescence, }}$ it can be selectively detected by the tracking head and used for position tracking. Unfortunately, the wavelength of this near-infrared light (800- to 900-nm range with a peak at $863 \mathrm{~nm}$ ) overlapped with the emission spectrum of ICG (the only Food and Drug Administration clinically approved near-infrared fluorescent dye; peak, $810 \mathrm{~nm}$ ). This meant that the tracking signal was also detected by the FC, causing a flickering interference when FI was performed in the near-infrared FI mode of the FC (Supplemental Fig. 1). Yet, this did not hinder navigation of the FC in the white-light setting. This flickering could be blocked efficiently by manually covering the tracking light sources. However, it did mean that the in-depth navigation information was temporarily lost. When the FC remained at the exact same distance, FI could be performed to evaluate if the sentinel node was fluorescent.

The spectral overlap between the tracking device and the fluorescent emission of ICG did not prevent us from demonstrating the potential of the hybrid navigation concept (Supplemental Fig. 1). However, for more routine use, the flickering issue needs to be solved. Here, the clinical availability of a spectrally different tracking light source or fluorescence tracer may provide outcome.

Because tracker placement appears to be a critical component for the navigation accuracy, much can be gained when this part of the procedure is improved. The $\mathrm{RT}_{\text {patient }}$ used in this study had to be removed and replaced during the transfer from the Department of Nuclear Medicine to the operation theater. To overcome this shortcoming, one might use a different method of coregistration, for example, by using multiple small metal balls that are not susceptible to deformation or by implementing the concept of intraoperative freehandSPECT (6-8). FreehandSPECT may help to correct for errors caused by tissue deformation as a result of surgical manipulation.

\section{DISCUSSION}

In this proof-of-concept study during open surgical SN biopsy procedures we demonstrated the feasibility of the hybrid navigator concept. This integrated approach provides an important step in the evolution of hybrid surgical guidance because it allows preoperative imaging information to be physically linked to FI in the intraoperative

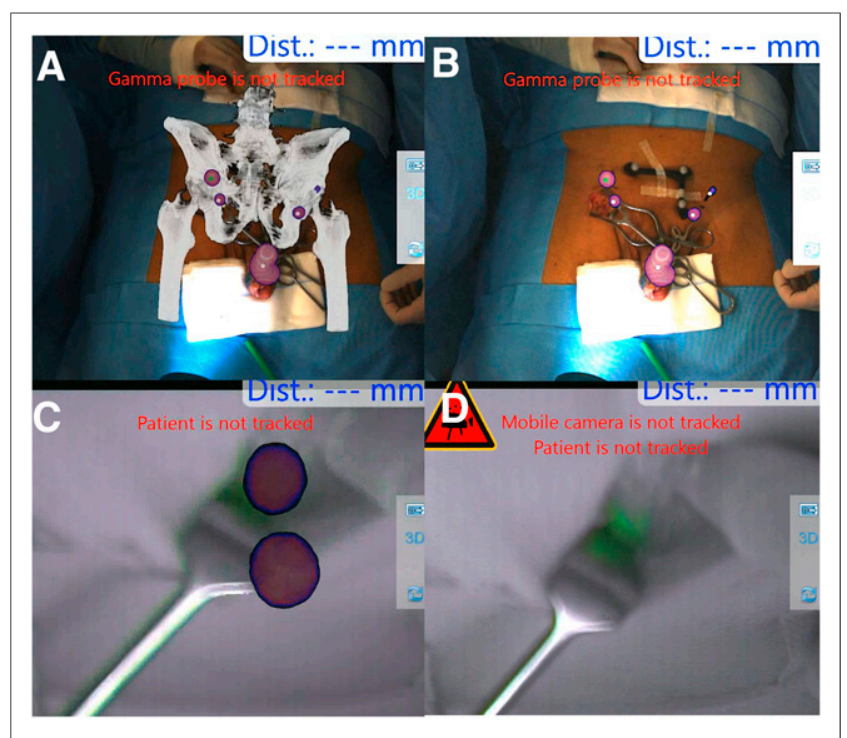

FIGURE 2. Navigation of the FC. Video signal of the overhead camera of navigation system allows projection of SPECT/CT (A) or SPECT (B) onto the patient. Bony structures (low-dose CT) and SNs/injection site (SPECT) are shown. (C) Overlay of the SPECT on near-infrared video signal of the FC showing 2 SNs. (D) Fluorescence imaging optically visualized the $\mathrm{SN}$ and thus allowed confirmation of FC navigation accuracy.

setting. Merging of modalities not only helps prevent a mismatch in findings, it also provides a step toward reducing space (e.g., in $\mathrm{m}^{2}$ they take up in the operation theater).

A fairly ideal evaluation setup was found in penile cancer patients as SNs were rather easy to locate because of their superficial location.

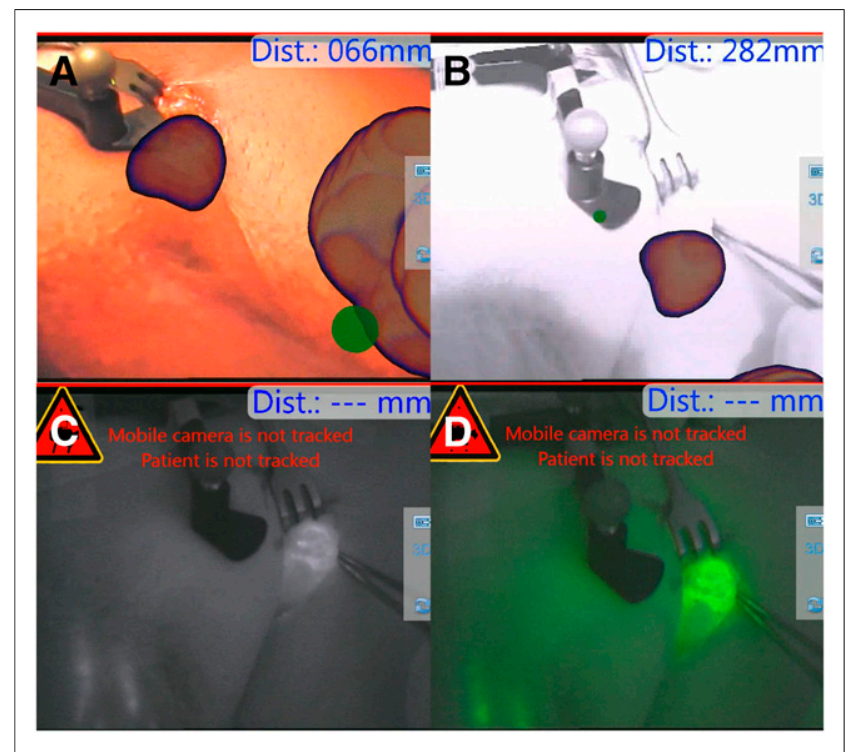

FIGURE 3. Fluorescence imaging with integrated overlay. (A) Preoperative overlay of the SPECT onto the patient from the perspective of the FC (white light imaging mode) showing the primary injected tumor and, close to the reference tracker, the SN. (B) Preoperative overlay of the SPECT onto the patient from the perspective of the FC (fluorescence imaging mode) illustrating the influence the flickering, caused by the tracking signal of the navigation device, has on the ability to visualize the fluorescence signal in the SN ( $C$ and $D$, in black-and-white or pseudocolored green, respectively). 
The main advantage of adding navigation in the hybrid surgical guidance concept was the improved directional feedback including depth estimation. As demonstrated in the current study, with the FC the accuracy of the navigation process could be confirmed or corrected. Similar to findings previously reported, the navigation error strongly depended on the accuracy with which we could replace the $\mathrm{RT}_{\text {patient }}$ during the surgical procedure $(7,8)$.

By including patients who undergo SN biopsy procedures in other anatomies, for example, in the head and neck or axilla, the value of this technique can be further evaluated. Alternative to navigating toward an $\mathrm{SN}$, one may also think of using this approach for hybrid tracer-based radioguided occult lesion localization (or for a seed-localization setup wherein hybrid marker seeds are applied) $(16,17)$. PET-based navigation in combination with intraoperative Cerenkov imaging could also provide an interesting extension (18).

\section{CONCLUSION}

Navigation-based integration of preoperative 3D SPECT/CT information with real-time FI provides an interesting next step in the hybrid surgical guidance concept. If the technology evolves further, its implementation may be extended to other (hybrid) tracers and anatomies.

\section{DISCLOSURE}

The costs of publication of this article were defrayed in part by the payment of page charges. Therefore, and solely to indicate this fact, this article is hereby marked "advertisement" in accordance with 18 USC section 1734. This work was partially supported by a Eurostars grant (Hybrid Navigator; grant E! 7555), an NWO-STW-VIDI grant (grant STW BGT11272), and a European Research Council under the European Union's Seventh Framework Program (FP7/20072013) grant (grant 2012-306890). The m-PDE FC used in this study was provided by Hamamatsu Photonics K.K. (Hamamatsu, Japan). SurgicEye (Munich, Germany) provided the prototype software for the navigation device. No other potential conflict of interest relevant to this article was reported.

\section{ACKNOWLEDGMENTS}

We gratefully acknowledge the surgical staff and the Department of Nuclear Medicine of the Netherlands Cancer Institute-Antoni van Leeuwenhoek Hospital for their support and assistance.

\section{REFERENCES}

1. van Leeuwen FWB, Hardwick JCH, van Erkel AR. Luminescence-based imaging approaches in the field of interventional molecular imaging. Radiology. 2015; 276:12-29.

2. Maurer T, Weirich G, Schottelius M, et al. Prostate-specific membrane antigenradioguided surgery for metastatic lymph nodes in prostate cancer. Eur Urol. 2015;68:530-534.

3. van den Berg NS. Valdés-Olmos R a, van der Poel HG, van Leeuwen FWB. Sentinel lymph node biopsy for prostate cancer: a hybrid approach. J Nucl Med. 2013;54:493-496.

4. Brouwer OR, Buckle T, Bunschoten A, et al. Image navigation as a means to expand the boundaries of fluorescence-guided surgery. Phys Med Biol. 2012;57:3123-3136.

5. van den Berg NS, Simon H, Kleinjan GH, et al. First-in-human evaluation of a hybrid modality that allows combined radio- and (near-infrared) fluorescence tracing during surgery. Eur J Nucl Med Mol Imaging. 2015;42:1639-1647.

6. Bluemel C, Schnelzer A, Okur A, et al. Freehand SPECT for image-guided sentinel lymph node biopsy in breast cancer. Eur J Nucl Med Mol Imaging. 2013;40:1656-1661.

7. Engelen T, Winkel BM, Rietbergen DD, et al. The next evolution in radioguided surgery: breast cancer related sentinel node localization using a freehandSPECTmobile gamma camera combination. Am J Nucl Med Mol Imaging. 2015;5:233-245.

8. Brouwer OR, van den Berg NS, Mathéron HM, et al. Feasibility of intraoperative navigation to the sentinel node in the groin using preoperatively acquired single photon emission computerized tomography data: transferring functional imaging to the operating room. $J$ Urol. 2014;192:1810-1816.

9. Freesmeyer M, Opfermann T, Winkens T. Hybrid integration of real-time US and freehand SPECT: proof of concept in patients with thyroid diseases. Radiology. 2014;271:856-861.

10. Brouwer OR, van den Berg NS, Mathéron HM, et al. A hybrid radioactive and fluorescent tracer for sentinel node biopsy in penile carcinoma as a potential replacement for blue dye. Eur Urol. 2014;65:600-609.

11. Sobin LH, Gospodarowicz MK, Wittekind C. TNM Classification of Malignant Tumours. 7th ed. Hoboken, NJ: Wiley-Blackwell. 2009.

12. KleinJan GH, Bunschoten A, van den Berg NS, et al. Fluorescence guided surgery and tracer-dose, fact or fiction? Eur J Nucl Med Mol Imaging. In press.

13. Bunschoten A, Buckle T, Kuil J, et al. Targeted non-covalent self-assembled nanoparticles based on human serum albumin. Biomaterials. 2012;33:867-875.

14. Zhang Z. A flexible new technique for camera calibration. IEEE Trans Pattern Anal Mach Intell. 2000;22:1330-1334.

15. van den Berg NS, Miwa M, Kleinjan GH, et al. (Near-infrared) fluorescence guided surgery under ambient light conditions, a next step to embedment of the technology in clinical routine. Ann Surg Oncol. In press.

16. van den Berg NS, Brouwer OR, Klop WMC, et al. Concomitant radio- and fluorescence-guided sentinel lymph node biopsy in squamous cell carcinoma of the oral cavity using ICG- ${ }^{99 \mathrm{~m}}$ Tc-nanocolloid. Eur J Nucl Med Mol Imaging. 2012;39:1128-1136.

17. Buckle T, Chin PTK, van den Berg NS, et al. Tumor bracketing and safety margin estimation using multimodal marker seeds: a proof of concept. J Biomed Opt. 2010;15:056021.

18. Thorek DLJ, Riedl CC, Grimm J. Clinical Cerenkov luminescence imaging of ${ }^{18}$ F-FDG. J Nucl Med. 2014;55:95-98. 